\title{
Can Hidden Variables Theories Meet Quantum Computation?
}

\author{
Koji Nagata ${ }^{1}$, Tadao Nakamura ${ }^{2}$ \\ ${ }^{1}$ Department of Physics, Korea Advanced Institute of Science and Technology, Daejeon, Korea \\ ${ }^{2}$ Department of Information and Computer Science, Keio University, Yokohama, Japan \\ Email: ko mi na@yahoo.co.jp, nakamura@pipelining.jp
}

Received 29 July 2015; accepted 21 August 2015; published 24 August 2015

Copyright (C) 2015 by authors and OALib.

This work is licensed under the Creative Commons Attribution International License (CC BY). http://creativecommons.org/licenses/by/4.0/

c) (i) Open Access

\begin{abstract}
We study the relation between hidden variables theories and quantum computation. We discuss an inconsistency between a hidden variables theory and controllability of quantum computation. To derive the inconsistency, we use the maximum value of the square of an expected value. We propose a solution of the problem by using new hidden variables theory. Also we discuss an inconsistency between hidden variables theories and the double-slit experiment as the most basic experiment in quantum mechanics. This experiment can be an easy detector to Pauli observable. We cannot accept hidden variables theories to simulate the double-slit experiment in a specific case. Hidden variables theories may not depicture quantum detector. This is a quantum measurement theoretical profound problem.
\end{abstract}

\section{Keywords}

Quantum Computer, Quantum Information Theory, Quantum Non Locality

Subject Areas: Applied Physics

\section{Introduction}

Quantum mechanics (cf. [1]-[6]) gives approximate and at times remarkably accurate numerical predictions. Much experimental data approximately fits to the quantum predictions for the past some 100 years. We do not doubt the correctness of quantum mechanics. Quantum mechanics also says new science with respect to information theory. The science is called the quantum information theory [6]. Therefore, quantum mechanics gives us very useful another theory in order to create new information science and to explain the handling of raw experimental data in our physical world.

As for the foundations of quantum mechanics, Leggett-type non-local variables theory [7] is experimentally investigated [8]-[10]. The experiments report that quantum mechanics does not accept Leggett-type 
non-local variables interpretation. As for the applications of quantum mechanics, implementation of a quantum algorithm to solve Deutsch's problem [11] on a nuclear magnetic resonance quantum computer is reported firstly [12]. Implementation of the Deutsch-Jozsa algorithm on an ion-trap quantum computer is also reported [13]. There are several attempts to use single-photon two-qubit states for quantum computing. Oliveira et al. implement Deutsch's algorithm with polarization and transverse spatial modes of the electromagnetic field as qubits [14]. Single-photon Bell states are prepared and measured [15]. Also the decoherence-free implementation of Deutsch's algorithm is reported by using such single-photon and by using two logical qubits [16]. More recently, a one-way based experimental implementation of Deutsch's algorithm is reported [17].

Given the fundamental studies and the application reports, we consider why quantum computer is faster than classical counterpart. It is essential to study the relation between hidden variables theory (classical theory) and quantum mechanics to investigate the quantum computation problem. So we address studying the relation between hidden variables theories and quantum computation.

We study the relation between hidden variables theories and quantum computation. The possible values of the pre-determined result of measurements are \pm 1 (in $\hbar / 2$ unit) in the original hidden variables theory. The reference frames are necessary to control a quantum state. We need controllability of quantum computation.

Let us consider controllability of quantum computation. We derive quantum proposition concerning a quantum expected value under an assumption about the existence of the orientation of reference frames in $N$ spin-1/2 systems. However, the original hidden variables theory violates the proposition with a magnitude that grows exponentially with the number of particles. To derive the inconsistency, we rely on the maximum value of the square of an hidden variables theoretical expected value. Therefore, we have to give up either the existence of the reference frames or the original hidden variables theory. The original hidden variables theory does not depicture physical phenomena using reference frames with a violation factor that grows exponentially with the number of particles.

The double-slit experiment is an illustration of wave-particle duality. In it, a beam of particles (such as photons) travels through a barrier with two slits removed. If one puts a detector screen on the other side, the pattern of detected particles shows interference fringes characteristic of waves; however, the detector screen responds to particles. The system exhibits the behaviour of both waves (interference patterns) and particles (dots on the screen).

If we modify this experiment so that one slit is closed, no interference pattern is observed. Thus, the state of both slits affects the final results. We can also arrange to have a minimally invasive detector at one of the slits to detect which slit the particle went through. When we do that, the interference pattern disappears. An analysis of a two-atom double-slit experiment based on environment-induced measurements is reported [18].

We assume an implementation of the double-slit experiment. There is a detector just after each slit. Thus interference figure does not appear, and we do not consider such a pattern. The possible values of the result of measurements are \pm 1 (in $\hbar / 2$ unit). If a particle passes one side slit, then the value of the result of measurement is +1 . If a particle passes through another slit, then the value of the result of measurement is -1 . This model is an easy detector model to Pauli observable.

We consider whether hidden variables theories meet an easy detector model to Pauli observable. We assume an implementation of the double-slit experiment. There is a detector just after each slit. We assume that a source of spin-carrying particles emits them in a state, which can be described as an eigenvector of Pauli observable $\sigma_{z}$. We consider a single expected value of Pauli observable $\sigma_{x}$ in the double-slit experiment. A wave function analysis says that the quantum expected value of it is zero. However, hidden variables theories can predict different value to the expected value of $\left\langle\sigma_{x}\right\rangle=0$. To derive the inconsistency, we use the maximum value of the square of an expected value. Hence, hidden variables theories do not meet the easy detector model as the whole.

Our paper is organized as follows.

In Section 2, we argue a hidden variables theory does not meet the reference frames.

In Section 3, we give a solution of the problem of the hidden variables theory. We find new hidden variables theory meets the reference frames.

In Section 4, we review the Deutsch-Jozsa algorithm using new hidden variables theory.

In Section 5, we discuss the relation between the double-slit experiment and hidden variables theories.

Section 6 concludes this paper. 


\section{A Hidden Variables Theory Does Not Meet the Reference Frames}

Assume that we have a set of $N$ spins $\frac{1}{2}$. Each of them is a spin-1/2 pure state lying in the $x-y$ plane. Let us assume that one source of $N$ uncorrelated spin-carrying particles emits them in a state, which can be described as a multi spin-1/2 pure uncorrelated state. Let us parameterize the settings of the $j$ th observer with a unit vector $\boldsymbol{n}_{j}$ (its direction along which the spin component is measured) with $j=1, \cdots, N$. One can introduce the "hidden variables" correlation function, which is the average of the product of the hidden results of measurement

$$
E_{\mathrm{HV}}\left(\boldsymbol{n}_{1}, \boldsymbol{n}_{2}, \cdots, \boldsymbol{n}_{N}\right)=\left\langle r\left(\boldsymbol{n}_{1}, \boldsymbol{n}_{2}, \cdots, \boldsymbol{n}_{N}\right)\right\rangle_{\text {avg }},
$$

where $r$ is the hidden result. We assume the value of $r$ is \pm 1 (in $(\hbar / 2)^{N}$ unit), which is obtained if the measurement directions are set at $\boldsymbol{n}_{1}, \boldsymbol{n}_{2}, \cdots, \boldsymbol{n}_{N}$.

Also one can introduce a quantum correlation function with the system in such a pure uncorrelated state

$$
E_{\mathrm{QM}}\left(\boldsymbol{n}_{1}, \boldsymbol{n}_{2}, \cdots, \boldsymbol{n}_{N}\right)=\operatorname{tr}\left[\rho \boldsymbol{n}_{1} \cdot \boldsymbol{\sigma} \otimes \boldsymbol{n}_{2} \cdot \boldsymbol{\sigma} \otimes \cdots \otimes \boldsymbol{n}_{N} \cdot \boldsymbol{\sigma}\right]
$$

where $\otimes$ denotes the tensor product, · the scalar product in $\mathbf{R}^{2}, \sigma=\left(\sigma_{x}, \sigma_{y}\right)$ is a vector of two Pauli operators, and $\rho$ is the pure uncorrelated state,

$$
\rho=\rho_{1} \otimes \rho_{2} \otimes \cdots \otimes \rho_{N}
$$

with $\rho_{j}=\left|\Psi_{j}\right\rangle\left\langle\Psi_{j}\right|$ and $\left|\Psi_{j}\right\rangle$ is a spin-1/2 pure state lying in the $x-y$ plane.

One can write the observable (unit) vector $\boldsymbol{n}_{j}$ in a plane coordinate system as follows:

$$
\boldsymbol{n}_{j}\left(\theta_{j}^{k_{j}}\right)=\cos \theta_{j}^{k_{j}} \boldsymbol{x}_{j}^{(1)}+\sin \theta_{j}^{k_{j}} \boldsymbol{x}_{j}^{(2)},
$$

where $\boldsymbol{x}_{j}^{(1)}=\boldsymbol{x}$ and $\boldsymbol{x}_{j}^{(2)}=\boldsymbol{y}$ are the Cartesian axes. Here, the angle $\theta_{j}^{k_{j}}$ takes two values (two-setting model):

$$
\theta_{j}^{1}=0, \theta_{j}^{2}=\frac{\pi}{2} .
$$

We derive a necessary condition to be satisfied by the quantum correlation function with the system in a pure uncorrelated state given in (2). In more detail, we derive the maximum value of the product of the quantum correlation function, $E_{\mathrm{QM}}$ given in (2), i.e., $\left\|E_{\mathrm{QM}}\right\|_{\max }^{2}$. We use the decomposition (4). We introduce simplified notations as

$$
T_{i_{1} i_{2} \cdots i_{N}}=\operatorname{tr}\left[\rho \boldsymbol{x}_{1}^{\left(i_{1}\right)} \cdot \boldsymbol{\sigma} \otimes \boldsymbol{x}_{2}^{\left(i_{2}\right)} \cdot \boldsymbol{\sigma} \otimes \cdots \otimes \boldsymbol{x}_{N}^{\left(i_{N}\right)} \cdot \boldsymbol{\sigma}\right]
$$

and

$$
\boldsymbol{c}_{j}=\left(c_{j}^{1}, c_{j}^{2}\right)=\left(\cos \theta_{j}^{k_{j}}, \sin \theta_{j}^{k_{j}}\right) .
$$

Then, we have

$$
\left\|E_{\mathrm{QM}}\right\|^{2}=\sum_{k_{1}=1}^{2} \cdots \sum_{k_{N}=1}^{2}\left(\sum_{i_{1}, \cdots, i_{N}=1}^{2} T_{i_{1} \cdots i_{N}} c_{1}^{i_{1}} \cdots c_{N}^{i_{N}}\right)^{2}=\sum_{i_{1}, \cdots, i_{N}=1}^{2} T_{i_{1} \cdots i_{N}}^{2} \leq 1,
$$

where we use the orthogonality relation $\sum_{k_{j}=1}^{2} c_{j}^{\alpha} c_{j}^{\beta}=\delta_{\alpha, \beta}$. The value of $\sum_{i_{1}, \cdots, i_{N}=1}^{2} T_{i_{1} \cdots i_{N}}^{2}$ is bounded as $\sum_{i_{1}, \cdots, i_{N}=1}^{2} T_{i_{1} \cdots i_{N}}^{2} \leq 1$. We have

$$
\prod_{j=1 i_{j}=1}^{N} \sum^{2}\left(\operatorname{tr}\left[\rho_{j} \boldsymbol{x}_{j}^{\left(i_{j}\right)} \cdot \boldsymbol{\sigma}\right]\right)^{2} \leq 1
$$

From the convex argument, all quantum separable states must satisfy the inequality (8). Therefore, it is a separability inequality. It is important that the separability inequality (8) is saturated iff $\rho$ is a multi spin-1/2 pure uncorrelated state such that, for every $j,\left|\Psi_{j}\right\rangle$ is a spin-1/2 pure state lying in the $x$-y plane. The reason of 
the inequality (8) is due to the following quantum inequality

$$
\sum_{i_{j}=1}^{2}\left(\operatorname{tr}\left[\rho_{j} \boldsymbol{x}_{j}^{\left(i_{j}\right)} \cdot \boldsymbol{\sigma}\right]\right)^{2} \leq 1
$$

The inequality (10) is saturated iff $\rho_{j}=\left|\Psi_{j}\right\rangle\left\langle\Psi_{j}\right|$ and $\left|\Psi_{j}\right\rangle$ is a spin-1/2 pure state lying in the $x-y$ plane. The inequality (8) is saturated iff the inequality (10) is saturated for every $j$. Thus we have the maximum possible value of the scalar product as a quantum proposition concerning the reference frames

$$
\left\|E_{\mathrm{QM}}\right\|_{\max }^{2}=1
$$

When the system is in such a multi spin-1/2 pure uncorrelated state.

On the other hand, a correlation function satisfies the hidden variables theory if it can be written as

$$
E_{\mathrm{HV}}\left(\boldsymbol{n}_{1}, \boldsymbol{n}_{2}, \cdots, \boldsymbol{n}_{N}\right)=\lim _{m \rightarrow \infty} \frac{\sum_{l=1}^{m} r\left(\boldsymbol{n}_{1}, \boldsymbol{n}_{2}, \cdots, \boldsymbol{n}_{N}, l\right)}{m}
$$

where $l$ denotes some hidden variable and $r$ is the hidden result of measurement of the dichotomic observables parameterized by the directions of $\boldsymbol{n}_{1}, \boldsymbol{n}_{2}, \cdots, \boldsymbol{n}_{N}$.

Assume the quantum correlation function with the system in a pure uncorrelated state given in (2) admits the hidden variables theory. One has the following proposition concerning the hidden variables theory

$$
E_{\mathrm{QM}}\left(\boldsymbol{n}_{1}, \boldsymbol{n}_{2}, \cdots, \boldsymbol{n}_{N}\right)=\lim _{m \rightarrow \infty} \frac{\sum_{l=1}^{m} r\left(\boldsymbol{n}_{1}, \boldsymbol{n}_{2}, \cdots, \boldsymbol{n}_{N}, l\right)}{m} .
$$

In what follows, we show that we cannot assign the truth value " 1 " for the proposition (13) concerning the hidden variables theory. We rely on the maximum value of the square of an expected value. Assume the proposition (13) is true. By changing the hidden variable $l$ into $l^{\prime}$, we have the same quantum expected value as follows

$$
E_{\mathrm{QM}}\left(\boldsymbol{n}_{1}, \boldsymbol{n}_{2}, \cdots, \boldsymbol{n}_{N}\right)=\lim _{m \rightarrow \infty} \frac{\sum_{l^{\prime}=1}^{m} r\left(\boldsymbol{n}_{1}, \boldsymbol{n}_{2}, \cdots, \boldsymbol{n}_{N}, l^{\prime}\right)}{m} .
$$

An important note here is that the value of the right-hand-side of (13) is equal to the value of the righthand-side of (14) because we only change the hidden variable.

We abbreviate $r\left(\boldsymbol{n}_{1}, \boldsymbol{n}_{2}, \cdots, \boldsymbol{n}_{N}, l\right)$ to $r(l)$ and $r\left(\boldsymbol{n}_{1}, \boldsymbol{n}_{2}, \cdots, \boldsymbol{n}_{N}, l^{\prime}\right)$ to $r\left(l^{\prime}\right)$.

We have

$$
\begin{aligned}
& \left\|E_{\mathrm{QM}}\right\|^{2}=\sum_{k_{1}=1}^{2} \cdots \sum_{k_{N}=1}^{2}\left(\lim _{m \rightarrow \infty} \frac{\sum_{l=1}^{m} r(l)}{m} \times \lim _{m \rightarrow \infty} \frac{\sum_{l^{\prime}=1}^{m} r\left(l^{\prime}\right)}{m}\right) \\
& \leq \sum_{k_{1}=1}^{2} \cdots \sum_{k_{N}=1}^{2}\left(\lim _{m \rightarrow \infty} \frac{\sum_{l=1}^{m}}{m} \times \lim _{m \rightarrow \infty} \frac{\sum_{l^{\prime}=1}^{m}}{m}\left|r(l) r\left(l^{\prime}\right)\right|\right) \\
& =\sum_{k_{1}=1}^{2} \cdots \sum_{k_{N}=1}^{2}\left(\lim _{m \rightarrow \infty} \frac{\sum_{l=1}^{m}}{m} \times \lim _{m \rightarrow \infty} \frac{\sum_{l^{\prime}=1}^{m}}{m}\right)=2^{N} .
\end{aligned}
$$

Here we use the fact

$$
\left|r(l) r\left(l^{\prime}\right)\right|=1
$$


Since the possible values of $r(l)$ are \pm 1 . The above inequality can be saturated because we have

$$
\begin{aligned}
& \{l \mid r(l)=1\}=\left\{l^{\prime} \mid r\left(l^{\prime}\right)=1\right\} \\
& \{l \mid r(l)=-1\}=\left\{l^{\prime} \mid r\left(l^{\prime}\right)=-1\right\} .
\end{aligned}
$$

Hence we derive the following proposition if we assign the truth value " 1 " for a hidden variables theory

$$
\left\|E_{\mathrm{QM}}\right\|_{\max }^{2}=2^{N} \text {. }
$$

Clearly, we cannot assign the truth value " 1 " for two propositions (11) (concerning the reference frames) and (18) (concerning the hidden variables theory), simultaneously, when the system is in a multiparticle pure uncorrelated state. Of course, each of them is a spin-1/2 pure state lying in the $x-y$ plane. Therefore, we are in the contradiction when the system is in such a multiparticle pure uncorrelated state. Thus, we cannot accept the validity of the proposition (13) (concerning the hidden variables theory) if we assign the truth value " 1 " for the proposition (11) (concerning the reference frames). In other words, the hidden variables theory does not reveal physical phenomena using reference frames. The reference frames are necessary to control a quantum state. Thus, the hidden variables theory does not reveal physical phenomena controlling a quantum state.

\section{Solution of the Problem of the Hidden Variables Theory}

In this section, we solve the contradiction presented in the previous section. We have the maximum possible value of the product as a quantum proposition concerning the reference frames

$$
\left\|E_{\mathrm{QM}}\right\|_{\max }^{2}=1
$$

when the system is in such a multi spin-1/2 pure uncorrelated state. On the other hand, one has the following proposition concerning the hidden variables theory

$$
\left\|E_{\mathrm{QM}}\right\|_{\max }^{2}=2^{N} .
$$

We cannot assign the truth value " 1 " for two propositions (19) (concerning the reference frames) and (20) (concerning the hidden variables theory), simultaneously, when the system is in a multiparticle pure uncorrelated state. Of course, each of them is a spin- $1 / 2$ pure state lying in the $x$ - $y$ plane. Therefore, we are in the contradiction when the system is in such a multiparticle pure uncorrelated state.

We introduce the following hypothesis:

Hypothesis: We assume the value of $r$ is $\pm \frac{1}{\sqrt{2^{N}}}\left(\right.$ in $(\hbar / 2)^{N}$ unit), which is obtained if the measurement directions are set at $\boldsymbol{n}_{1}, \boldsymbol{n}_{2}, \cdots, \boldsymbol{n}_{N}$.

When we accept this hypothesis, the proposition (20) (concerning the hidden variables theory) becomes the following new proposition concerning other hidden variables theory (two-setting model)

$$
\left\|E_{\mathrm{QM}}\right\|_{\max }^{2}=1 \text {. }
$$

We can assign the truth value " 1 " for both two propositions (19) (concerning the reference frames) and (21) (concerning other hidden variables theory), simultaneously, when the system is in a multiparticle pure uncorrelated state. Of course, each of them is a spin- $1 / 2$ pure state lying in the $x-y$ plane. Therefore, we are not in the contradiction when the system is in such a multiparticle pure uncorrelated state. Hence, we solve the contradiction presented in the previous section by changing the value of the result of pre-determined measurements. Our solution is equivalent to changing Planck's constant $(\hbar)$ to the new constant $(\hbar / \sqrt{2})$.

\section{The Deutsch-Jozsa Algorithm Using New Hidden Variables Theory}

The earliest quantum algorithm, the Deutsch-Jozsa algorithm, is representative to show that quantum computation is faster than classical counterpart with a magnitude that grows exponentially with the number of qubits.

Let us follow the argumentation presented in [6]. The application, known as Deutsch's problem, may be 
described as the following game. Alice, in Amsterdam, selects a number $x$ from 0 to $2^{N}-1$, and mails it in a letter to Bob, in Boston. Bob calculates the value of some function

$$
f:\left\{0, \cdots, 2^{N}-1\right\} \rightarrow\{0,1\}
$$

and replies with the result, which is either 0 or 1 . Now, Bob has promised to use a function $f$ which is of one of two kinds; either the value of $f(x)$ is constant for all values of $x$, or else the value of $f(x)$ is balanced, that is, equal to 1 for exactly half of all the possible $x$, and 0 for the other half. Alice's goal is to determine with certainty whether Bob has chosen a constant or a balanced function, corresponding with him as little as possible. How fast can she succeed?

In the classical case, Alice may only send Bob one value of $x$ in each letter. At worst, Alice will need to query Bob at least

$$
2^{N} / 2+1
$$

times, since she may receive $2^{N} / 2$ os before finally getting a 1 , telling her that Bob's function is balanced. The best deterministic classical algorithm she can use therefore requires $2^{N} / 2+1$ queries. Note that in each letter, Alice sends Bob $N$ bits of information. Furthermore, in this example, physical distance is being used to artificially elevate the cost of calculating $f(x)$, but this is not needed in the general problem, where $f(x)$ may be inherently difficult to calculate.

If Bob and Alice were able to exchange qubits, instead of just classical bits, and if Bob agreed to calculate $f(x)$ using a unitary transformation $U_{f}$, then Alice could achieve her goal in just one correspondence with Bob, using the following algorithm.

Alice has an $N$ qubit register to store her query in, and a single qubit register which she will give to Bob, to store the answer in. She begins by preparing both her query and answer registers in a superposition state. Bob will evaluate $f(x)$ using quantum parallelism and leave the result in the answer register. Alice then interferes states in the superposition using a Hadamard transformation (a unitary transformation),

$$
H=\left(\sigma_{x}+\sigma_{z}\right) / \sqrt{2},
$$

on the query register, and finishes by performing a suitable measurement to determine whether $f$ was constant or balanced.

Let us follow the quantum states through this algorithm. The input state is

$$
\left|\psi_{0}\right\rangle=|0\rangle^{\otimes N}|1\rangle .
$$

Here the query register describes the state of $N$ qubits all prepared in the

state. After the Hadamard transformation on the query register and the Hadamard gate on the answer register we have

$$
\left|\psi_{1}\right\rangle=\sum_{x \in\{0,1\}^{N}} \frac{|x\rangle}{\sqrt{2^{N}}}\left[\frac{|0\rangle-|1\rangle}{\sqrt{2}}\right] .
$$

The query register is now a superposition of all values, and the answer register is in an evenly weighted superposition of

And

$|1\rangle$.

Next, the function $f$ is evaluated (by Bob) using

$$
U_{f}:|x, y\rangle \rightarrow|x, y \oplus f(x)\rangle,
$$

giving 


$$
\left|\psi_{2}\right\rangle= \pm \sum_{x} \frac{(-1)^{f(x)}|x\rangle}{\sqrt{2^{N}}}\left[\frac{|0\rangle-|1\rangle}{\sqrt{2}}\right] .
$$

Here

$$
y \oplus f(x)
$$

is the bitwise XOR (exclusive OR) of $y$ and $f(x)$. Alice now has a set of qubits in which the result of Bob's function evaluation is stored in the amplitude of the qubit superposition state. She now interferes terms in the superposition using a Hadamard transformation on the query register. To determine the result of the Hadamard transformation it helps to first calculate the effect of the Hadamard transformation on a state

$$
|x\rangle \text {. }
$$

By checking the cases $x=0$ and $x=1$ separately we see that for a single qubit

$$
H|x\rangle=\sum_{z}(-1)^{x z}|z\rangle / \sqrt{2} .
$$

Thus

$$
H^{\otimes N}\left|x_{1}, \cdots, x_{N}\right\rangle=\frac{\sum_{z_{1}, \cdots, z_{N}}(-1)^{x_{1} z_{1}+\cdots+x_{N} z_{N}}\left|z_{1}, \cdots, z_{N}\right\rangle}{\sqrt{2^{N}}} .
$$

This can be summarized more succinctly in the very useful equation

$$
H^{\otimes N}|x\rangle=\frac{\sum_{z}(-1)^{x \cdot z}|z\rangle}{\sqrt{2^{N}}},
$$

where

$$
x \cdot z
$$

is the bitwise inner product of $x$ and $z$, modulo 2. Using this equation and (31) we can now evaluate $\left|\psi_{3}\right\rangle$,

$$
\left|\psi_{3}\right\rangle= \pm \sum_{z} \sum_{x} \frac{(-1)^{x \cdot z+f(x)}|z\rangle}{\sqrt{2^{N}}}\left[\frac{|0\rangle-|1\rangle}{\sqrt{2}}\right] .
$$

Alice now observes the query register. Note that the absolute value of the amplitude for the state

$$
|0\rangle^{\otimes N}
$$

Is

$$
\sum_{x}(-1)^{f(x)} / 2^{N} .
$$

Let's look at the two possible cases- $f$ constant and $f$ balanced-to discern what happens. In the case where $f$ is constant the absolute value of the amplitude for

$$
|0\rangle^{\otimes N}
$$

is +1 . Because

$$
\left|\psi_{3}\right\rangle
$$

is of unit length it follows that all the other amplitudes must be zero, and an observation will yield

$$
\left(+\frac{1}{\sqrt{2}}\right)
$$


times for all $N$ qubits in the query register. Thus, global measurement outcome is

$$
\left(+\frac{1}{\sqrt{2^{N}}}\right) \text {. }
$$

If $f$ is balanced then the positive and negative contributions to the absolute value of the amplitude for

$$
|0\rangle^{\otimes N}
$$

cancel, leaving an amplitude of zero, and a measurement must yield a result other than

$$
+\frac{1}{\sqrt{2}}
$$

that is,

$$
-\frac{1}{\sqrt{2}}
$$

on at least one qubit in the query register. Summarizing, if Alice measures all $\left(+\frac{1}{\sqrt{2}}\right) \mathrm{s}$ and global measurement outcome is $\left(+\frac{1}{\sqrt{2^{N}}}\right)$ the function is constant; otherwise the function is balanced.

We notice that the difference between $+\frac{1}{\sqrt{2^{N}}}$ and $-\frac{1}{\sqrt{2^{N}}}$ is approximately zero when $N \gg 1$. We question if the Deutsch-Jozsa algorithm in the macroscopic scale is possible or not. This question is open problem.

We see the measurement outcome is predetermined. This is classical situation. We can see the result of the Deutsch-Jozsa algorithm classically. And an input state violates non local realism [19]. This is quantum theoretical situation. The Deutsch-Jozsa algorithm is performed in the arrow of time. The arrow of time goes from quantum theory to classical theory. This physical situation is similar to the quantum decoherence. We may say the Deutsch-Jozsa algorithm is physical.

\section{The Double-Slit Experiment and Hidden Variables Theories}

In this section, we consider the relation between the double-slit experiment and the original hidden variables theory. We assume an implementation of the double-slit experiment. There is a detector just after each slit. Thus interference figure does not appear, and we do not consider such a pattern. The possible values of the result of measurements are \pm 1 (in $\hbar / 2$ unit). If a particle passes one side slit, then the value of the result of measurement is +1 . If a particle passes through another slit, then the value of the result of measurement is -1 .

\subsection{A Wave Function Analysis}

Let $\left(\sigma_{z}, \sigma_{x}\right)$ be Pauli vector. We assume that a source of spin-carrying particles emits them in a state $|\psi\rangle$, which can be described as an eigenvector of Pauli observable $\sigma_{z}$. We consider a quantum expected value $\left\langle\sigma_{x}\right\rangle$ as

$$
\left\langle\sigma_{x}\right\rangle=\left\langle\psi\left|\sigma_{x}\right| \psi\right\rangle=0
$$

The above quantum expected value is zero if we consider only a wave function analysis.

We derive a necessary condition for the quantum expected value for the system in the pure spin- $1 / 2$ state $|\psi\rangle$ given in (48). We derive the possible value of the product $\left\langle\sigma_{x}\right\rangle \times\left\langle\sigma_{x}\right\rangle=\left\langle\sigma_{x}\right\rangle^{2} .\left\langle\sigma_{x}\right\rangle$ is the quantum expected value given in (48). We have

$$
\left\langle\sigma_{x}\right\rangle^{2}=0
$$

Thus, 


$$
\left\langle\sigma_{x}\right\rangle^{2} \leq 0
$$

We derive the following proposition

$$
\left(\left\langle\sigma_{x}\right\rangle^{2}\right)_{\max }=0
$$

\subsection{The Original Hidden Variables Theory}

On the other hand, a mean value $E$ admits the hidden variables theory if it can be written as

$$
E=\frac{\sum_{l=1}^{m} r_{l}\left(\sigma_{x}\right)}{m}
$$

where $l$ denotes some hidden variable and $r$ is the hidden result of measurement of the Pauli observable $\sigma_{x}$. We assume the value of $r$ is \pm 1 (in $\hbar / 2$ unit).

Assume the quantum mean value with the system in an eigenvector $(|\psi\rangle)$ of Pauli observable $\sigma_{z}$ given in (48) admits the hidden variables theory. One has the following proposition concerning the hidden variables theory

$$
\left\langle\sigma_{x}\right\rangle(m)=\frac{\sum_{l=1}^{m} r_{l}\left(\sigma_{x}\right)}{m} .
$$

We can assume as follows by Strong Law of Large Numbers,

$$
\left\langle\sigma_{x}\right\rangle(+\infty)=\left\langle\sigma_{x}\right\rangle=\left\langle\psi\left|\sigma_{x}\right| \psi\right\rangle .
$$

In what follows, we show that we cannot assign the truth value "1" for the proposition (53) concerning the hidden variables theory. We rely on the maximum value of the square of a mean value.

Assume the proposition (53) is true. By changing the hidden variable $l$ into $l^{\prime}$, we have the same quantum mean value as follows

$$
\left\langle\sigma_{x}\right\rangle(m)=\frac{\sum_{I^{\prime}=1}^{m} r_{l^{\prime}}\left(\sigma_{x}\right)}{m} .
$$

An important note here is that the value of the right-hand-side of (53) is equal to the value of the righthand-side of (55) because we only change the hidden variable. We have

$$
\left\langle\sigma_{x}\right\rangle(m) \times\left\langle\sigma_{x}\right\rangle(m)=\frac{\sum_{l=1}^{m} r_{l}\left(\sigma_{x}\right)}{m} \times \frac{\sum_{l^{\prime}=1}^{m} r_{l^{\prime}}\left(\sigma_{x}\right)}{m} \leq \frac{\sum_{l=1}^{m}}{m} \times \frac{\sum_{l^{\prime}=1}^{m}}{m}\left|r_{l}\left(\sigma_{x}\right) r_{l^{\prime}}\left(\sigma_{x}\right)\right|=\frac{\sum_{l=1}^{m}}{m} \times \frac{\sum_{l^{\prime}=1}^{m}}{m}=1 .
$$

Here we use the fact

$$
\left|r_{l}\left(\sigma_{x}\right) r_{l^{\prime}}\left(\sigma_{x}\right)\right|=1
$$

since the possible values of $r_{l}\left(\sigma_{x}\right)$ are \pm 1 . The above inequality can be saturated because we have

$$
\begin{aligned}
& \left\{l \mid r_{l}\left(\sigma_{x}\right)=1\right\}=\left\{l^{\prime} \mid r_{l^{\prime}}\left(\sigma_{x}\right)=1\right\} \\
& \left\{l \mid r_{l}\left(\sigma_{x}\right)=-1\right\}=\left\{l^{\prime} \mid r_{l^{\prime}}\left(\sigma_{x}\right)=-1\right\} .
\end{aligned}
$$

Hence we derive the following proposition if we assign the truth value " 1 " for a hidden variables theory

$$
\left(\left\langle\sigma_{x}\right\rangle(m) \times\left\langle\sigma_{x}\right\rangle(m)\right)_{\max }=1 .
$$

From Strong Law of Large Numbers, we have

$$
\left(\left\langle\sigma_{x}\right\rangle \times\left\langle\sigma_{x}\right\rangle\right)_{\max }=1 .
$$


Hence we derive the following proposition concerning the hidden variables theory

$$
\left(\left\langle\sigma_{x}\right\rangle^{2}\right)_{\max }=1 .
$$

We do not assign the truth value "1" for two propositions (51) (concerning a wave function analysis) and (61) (concerning the hidden variables theory), simultaneously. We are in the contradiction.

We cannot accept the validity of the proposition (53) (concerning the hidden variables theory) if we assign the truth value " 1 " for the proposition (51) (concerning a wave function analysis). In other words, we cannot accept the hidden variables theory to simulate the detector model for spin observable $\sigma_{x}$.

\subsection{New Hidden Variables Theory}

A mean value $E$ admits new hidden variables theory if it can be written as

$$
E=\frac{\sum_{l=1}^{m} r_{l}\left(\sigma_{x}\right)}{m}
$$

where $l$ denotes some hidden variable and $r$ is the hidden result of measurement of the Pauli observable $\sigma_{x}$. We assume the value of $r$ is $\pm 1 / \sqrt{2}$ (in $\hbar / 2$ unit).

Assume the quantum mean value with the system in an eigenvector $(|\psi\rangle)$ of Pauli observable $\sigma_{z}$ given in (48) admits new hidden variables theory. One has the following proposition concerning new hidden variables theory

$$
\left\langle\sigma_{x}\right\rangle(m)=\frac{\sum_{l=1}^{m} r_{l}\left(\sigma_{x}\right)}{m} .
$$

We can assume as follows by Strong Law of Large Numbers,

$$
\left\langle\sigma_{x}\right\rangle(+\infty)=\left\langle\sigma_{x}\right\rangle=\left\langle\psi\left|\sigma_{x}\right| \psi\right\rangle .
$$

In what follows, we show that we cannot assign the truth value " 1 " for the proposition (63) concerning new hidden variables theory. We rely on the maximum value of the square of a mean value.

Assume the proposition (63) is true. By changing the hidden variable $l$ into $l^{\prime}$, we have the same quantum mean value as follows

$$
\left\langle\sigma_{x}\right\rangle(m)=\frac{\sum_{I^{\prime}=1}^{m} r_{l^{\prime}}\left(\sigma_{x}\right)}{m} .
$$

An important note here is that the value of the right-hand-side of (63) is equal to the value of the right-handside of (65) because we only change the hidden variable. We have

$$
\left\langle\sigma_{x}\right\rangle(m) \times\left\langle\sigma_{x}\right\rangle(m)=\frac{\sum_{l=1}^{m} r_{l}\left(\sigma_{x}\right)}{m} \times \frac{\sum_{l^{\prime}=1}^{m} r_{l^{\prime}}\left(\sigma_{x}\right)}{m} \leq \frac{\sum_{l=1}^{m}}{m} \times \frac{\sum_{l^{\prime}=1}^{m}}{m}\left|r_{l}\left(\sigma_{x}\right) r_{l^{\prime}}\left(\sigma_{x}\right)\right|=\frac{\sum_{l=1}^{m}}{m} \times \frac{\sum_{l^{\prime}=1}^{m}}{m}(1 / 2)=1 / 2 .
$$

Here we use the fact

$$
\left|r_{l}\left(\sigma_{x}\right) r_{l^{\prime}}\left(\sigma_{x}\right)\right|=1 / 2
$$

since the possible values of $r_{l}\left(\sigma_{x}\right)$ are $\pm 1 / \sqrt{2}$. The above inequality can be saturated because we have

$$
\begin{aligned}
& \left\{l \mid r_{l}\left(\sigma_{x}\right)=1 / \sqrt{2}\right\}=\left\{l^{\prime} \mid r_{l^{\prime}}\left(\sigma_{x}\right)=1 / \sqrt{2}\right\} \\
& \left\{l \mid r_{l}\left(\sigma_{x}\right)=-1 / \sqrt{2}\right\}=\left\{l^{\prime} \mid r_{l^{\prime}}\left(\sigma_{x}\right)=-1 / \sqrt{2}\right\} .
\end{aligned}
$$

Hence we derive the following proposition if we assign the truth value " 1 " for new hidden variables theory

$$
\left(\left\langle\sigma_{x}\right\rangle(m) \times\left\langle\sigma_{x}\right\rangle(m)\right)_{\max }=1 / 2
$$


From Strong Law of Large Numbers, we have

$$
\left(\left\langle\sigma_{x}\right\rangle \times\left\langle\sigma_{x}\right\rangle\right)_{\max }=1 / 2 .
$$

Hence we derive the following proposition concerning new hidden variables theory

$$
\left(\left\langle\sigma_{x}\right\rangle^{2}\right)_{\max }=1 / 2
$$

We do not assign the truth value " 1 " for two propositions (51) (concerning a wave function analysis) and (71) (concerning new hidden variables theory), simultaneously. We are in the contradiction.

We cannot accept the validity of the proposition (63) (concerning new hidden variables theory) if we assign the truth value " 1 " for the proposition (51) (concerning a wave function analysis). In other words, we cannot accept new hidden variables theory to simulate the detector model for spin observable $\sigma_{x}$.

\section{Conclusions}

In conclusion, we have studied the relation between a hidden variables theory and quantum computation. The possible values of the pre-determined result of measurements have been \pm 1 (in $\hbar / 2$ unit). The reference frames have been necessary to control a quantum state.

We have derived some proposition concerning a quantum expected value under an assumption about the existence of the orientation of reference frames in $N$ spin-1/2 systems. However, the hidden variables theory has violated the proposition with a magnitude that grows exponentially with the number of particles. Therefore, we have had to give up either the existence of the reference frames or the hidden variables theory. The hidden variables theory does not have depictured physical phenomena using reference frames with a violation factor that grows exponentially with the number of particles.

We have proposed a solution of the problem. Our solution has been equivalent to changing Planck's constant $(\hbar)$ to a new constant $(\hbar / \sqrt{2})$. The Deutsch-Jozsa algorithm has been performed in the arrow of time. The arrow of time has gone from quantum theory to classical theory. This physical situation had been similar to the quantum decoherence.

We may have said the Deutsch-Jozsa algorithm is physical. Also we have discussed the fact that both the original hidden variables theory and new hidden variables theory do not meet an easy detector model to a single Pauli observable. Hidden variables theories may not depicture quantum detector. This is a quantum measurement theoretical profound problem.

\section{Acknowledgements}

We thank Professor Weinstein for valuable discussions.

\section{References}

[1] von Neumann, J. (1955) Mathematical Foundations of Quantum Mechanics. Princeton University Press, Princeton.

[2] Feynman, R.P., Leighton, R.B. and Sands, M. (1965) Lectures on Physics. Volume 3, Quantum Mechanics, AddisonWesley Publishing Company.

[3] Redhead, M. (1989) Incompleteness, Nonlocality, and Realism. 2nd Edition, Clarendon Press, Oxford.

[4] Peres, A. (1993) Quantum Theory: Concepts and Methods. Kluwer Academic, Dordrecht.

[5] Sakurai, J.J. (1995) Modern Quantum Mechanics. Addison-Wesley Publishing Company.

[6] Nielsen, M.A. and Chuang, I.L. (2000) Quantum Computation and Quantum Information. Cambridge University Press, Cambridge.

[7] Leggett, A.J. (2003) Nonlocal Hidden-Variable Theories and Quantum Mechanics: An Incompatibility Theorem. Foundations of Physics, 33, 1469-1493. http://dx.doi.org/10.1023/A:1026096313729

[8] Gröblacher, S., Paterek, T., Kaltenbaek, R., Brukner, Č., Żukowski, M., Aspelmeyer, M. and Zeilinger, A. (2007) An Experimental Test of Non-Local Realism. Nature (London), 446, 871-875. http://dx.doi.org/10.1038/nature05677

[9] Paterek, T., Fedrizzi, A., Gröblacher, S., Jennewein, T., Żukowski, M., Aspelmeyer, M. and Zeilinger, A. (2007) Experimental Test of Nonlocal Realistic Theories without the Rotational Symmetry Assumption. Physical Review Letters, 99, Article ID: 210406. http://dx.doi.org/10.1103/PhysRevLett.99.210406 
[10] Branciard, C., Ling, A., Gisin, N., Kurtsiefer, C., Lamas-Linares, A. and Scarani, V. (2007) Experimental Falsification of Leggett’s Nonlocal Variable Model. Physical Review Letters, 99, Article ID: 210407. http://dx.doi.org/10.1103/PhysRevLett.99.210407

[11] Deutsch, D. (1985) Quantum Theory, the Church-Turing Principle and the Universal Quantum Computer. Proceedings of the Royal Society of London. Series A, 400, 97. http://dx.doi.org/10.1098/rspa.1985.0070

[12] Jones, J.A. and Mosca, M. (1998) Implementation of a Quantum Algorithm on a Nuclear Magnetic Resonance Quantum Computer. The Journal of Chemical Physics, 109, 1648. http://dx.doi.org/10.1063/1.476739

[13] Gulde, S., Riebe, M., Lancaster, G.P.T., Becher, C., Eschner, J., Häffner, H., Schmidt-Kaler, F., Chuang, I.L. and Blatt, R. (2003) Implementation of the Deutsch-Jozsa Algorithm on an Ion-Trap Quantum Computer. Nature, 421, 48-50. http://dx.doi.org/10.1038/nature01336

[14] de Oliveira, A.N., Walborn, S.P. and Monken, C.H. (2005) Implementing the Deutsch Algorithm with Polarization and Transverse Spatial Modes. Journal of Optics B: Quantum and Semiclassical Optics, 7, 288-292. http://dx.doi.org/10.1088/1464-4266/7/9/009

[15] Kim, Y.-H. (2003) Single-Photon Two-Qubit Entangled States: Preparation and Measurement. Physical Review A, 67, Article ID: 040301(R).

[16] Mohseni, M., Lundeen, J.S., Resch, K.J. and Steinberg, A.M. (2003) Experimental Application of Decoherence-Free Subspaces in an Optical Quantum-Computing Algorithm. Physical Review Letters, 91, Article ID: 187903. http://dx.doi.org/10.1103/PhysRevLett.91.187903

[17] Tame, M.S., Prevedel, R., Paternostro, M., Böhi, P., Kim, M.S. and Zeilinger, A. (2007) Experimental Realization of Deutsch’s Algorithm in a One-Way Quantum Computer. Physical Review Letters, 98, Article ID: 140501. http://dx.doi.org/10.1103/PhysRevLett.98.140501

[18] Schon, C. and Beige, A. (2001) Analysis of a Two-Atom Double-Slit Experiment Based on Environment-Induced Measurements. Physical Review A, 64, Article ID: 023806. http://dx.doi.org/10.1103/PhysRevA.64.023806

[19] Nagata, K. (2010) Implementation of the Deutsch-Jozsa Algorithm Violates Nonlocal Realism. The European Physical Journal D, 56, 441-444. http://dx.doi.org/10.1140/epjd/e2009-00303-6 